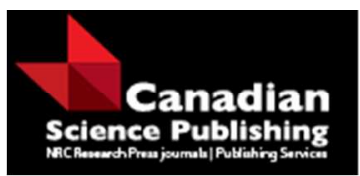

Canadian Journal of Forest Research Revue canadienne de recherche forestière

\title{
Global Sensitivity Analysis for the Rothermel Model Based on High Dimensional Model Representation
}

\begin{tabular}{|r|l|}
\hline Journal: & Canadian Journal of Forest Research \\
\hline Manuscript ID & cjfr-2015-0148.R1 \\
\hline Manuscript Type: & Article \\
\hline Complete List of Authors: & $\begin{array}{l}\text { Liu, Yaning; Lawrence Berkeley National Laboratory, } \\
\text { Hussaini, Yousuff; Florida State University, Mathematics } \\
\text { Okten, Giray; Florida State University, Mathematics Department }\end{array}$ \\
\hline Keyword: & $\begin{array}{l}\text { Rothermel fire spread model, Global sensitivity analysis, High dimensional } \\
\text { model representation, Monte Carlo methods, ANOVA }\end{array}$ \\
\hline \multicolumn{2}{|c}{} \\
\hline \multicolumn{2}{|c|}{} \\
\hline
\end{tabular}

\section{SCHOLARONE ${ }^{m}$}

Manuscripts 
Global Sensitivity Analysis for the Rothermel Model Based on High

Dimensional Model Representation

\section{Yaning Liu}

Earth Sciences Division, Lawrence Berkeley National Laboratory, Berkeley, CA

94720, USA. Email: yaningliu@lbl.gov

M. Yousuff Hussaini

Department of Mathematics, Florida State University, Tallahassee, FL 32306-

4510, USA. Email: yousuff@,fsu.edu

Giray Ökten ${ }^{*}$

Department of Mathematics, Florida State University, Tallahassee, FL 32306-

4510, USA. Email: okten@math.fsu.edu

* Corresponding author. Phone: (850) 644-8713 Fax: (850) 644-4053 


\begin{abstract}
Rothermel's wildland surface fire spread model is widely used in North America. The model outputs depend on a number of input parameters, which can be broadly categorized as fuel model, fuel moisture, terrain and wind parameters. Due to the inevitable presence of uncertainty in the input parameters, the sensitivity of the model output to a given input parameter can be very useful for understanding and controlling the sources of parametric uncertainty. Instead of obtaining the local sensitivity indices, we perform a global sensitivity analysis that considers the synchronous changes of parameters in their respective ranges. The global sensitivity indices corresponding to different parameter groups are computed by constructing the truncated ANOVA-high dimensional model representation for the model outputs with a polynomial expansion approach. We apply global sensitivity analysis to six standard fuel models, namely, short grass, tall grass, chaparral, hardwood litter, timber and light logging slash. Our sensitivity results show similarities as well as differences between fuel models. For example, the sensitivities of the input parameters fuel depth, low heat content, and wind, are large in all fuel models, and as high as $85 \%$ of the total model variance in the fuel model light logging slash. On the other hand, the fuel depth explains around $40 \%$ of the total variance in the fuel model light logging slash, but only $12 \%$ for the fuel model short grass. The quantification of the importance of parameters across fuel models helps identify the parameters for which additional resources should be used to lower their uncertainty, leading to effective fire management.
\end{abstract}

Keywords: Rothermel fire spread model, global sensitivity analysis, high dimensional model representation, Monte Carlo methods, ANOVA 


\section{Introduction}

Wildland fire management requires an understanding of fire behaviors. Predicting fire behavior can be based solely on experience, which requires the predictor to have a profound comprehension of the interrelation of fire, topography, fuel and weather, which are collectively referred to as the fire environment (Pyne et al. 1996). Fire can present a variety of behaviors and can be affected by a wide range of environmental factors whose variations can lead to both drastic changes of fire behaviors that easily draw predictors' attention and moderate ones that are hard to notice but may be crucial to the growth of the fire. Alternatively, fire modeling attempts to simulate fire behaviors in a repeatable way and has been playing an increasingly vital role in aiding fire management activities, especially with the development of rapid computing facilities and algorithms.

Fire models are in general classified as statistical models (McMaster 1973), empirical models (Rothermel 1972), physical models (Albini 1986), and atmosphere-fire models (Linn et al. 2000; Clark 2004). On account of the varying computational complexities, one model may be more suitable for one task than for the others. For example, empirical models are often used for prediction, while physical and atmosphere-fire models are used in post-processing. To facilitate the fulfillment of various fire management needs, various fire models have been incorporated in software systems such as FARSITE (Finney 2004) and BehavePlus (Andrews 2007).

Rothermel's surface fire spread model (Rothermel 1972) is a widely used wildland fire model in North America. The model is categorized as "semi-empirical" since it was developed using the principle of conservation of energy, as well as experiments. There have been several 
variants of the Rothermel model in the literature that modify the modeling of certain terms (see Albini 1976, Wilson1990, Catchpole and Catchpole 1991). Also, Sandberg et al. (2007) extends the original model so that it can predict fire behaviors for more complex fuelbeds.

The Rothermel model involves a wide range of input parameters, such as fuel type, fuel moisture contents, terrain and wind related variables. These parameters are subject to uncertainties, due to model error, modeling error, and data error. As a result, the model outputs are also uncertain. Jimenez et al. (2007, 2008) addressed the uncertainty quantification of the Rothermel model with efficient Monte Carlo sampling methods. Another important question is the sensitivity analysis of the model. Sensitivity analysis quantifies the amount of uncertainty in the output contributed by an input parameter (or, group of parameters). Sensitivity information is of critical importance in wildfire management since it allows for allocation of resources to control the factors that are more important for fire behavior than others. Sensitivity analysis can be local, in the sense that the rate of change of the model output with respect to a certain parameter at some given point is taken as the measure for sensitivity. On the other hand, global sensitivity analysis (GSA) completely explores the parameter space, considering interactions among parameters.

The variance-based Sobol' GSA (Sobol' 1993, 2001) is a popular method for computing the sensitivities of a model. The variance of each ANOVA-high dimensional model representation (HDMR) component is used as the measure of its importance. Salvador et al. (2001) studied Sobol' sensitivity analysis of the Rothermel model for Mediterranean shrublands. The Sobol' indices were computed by estimating high dimensional conditional expectations. Liu et al. (2015) used sensitivity analysis to reduce the number of model parameters, and applied randomized quasi-Monte Carlo methods together with a control 
variate technique to quantify parametric uncertainties for the reduced model. In most physical systems, noticeable high orders of parameter interaction are rarely seen (Rabitz et al. 1999), which means considering only low order parameter interactions can sufficiently represent a model. Based on this observation, we propose using an orthogonal polynomial expansion for the truncated ANOVA-HDMR of the Rothermel model. The Sobol' indices can be obtained directly from the expansion coefficients. We perform Sobol' sensitivity analysis for six fuel models selected from the 13 standard fuel models (Anderson 1982, Albini 1976), namely short grass, tall grass, chaparral, hardwood litter, timber, and light logging slash. We observe significant differences in the sensitivity patterns for different types of fuel, underlying the importance of sensitivity analysis to determine the significant parameters of the model.

\section{Formulation of the Rothermel Model}

The Rothermel model consists of more than 80 highly nonlinear algebraic equations. The main outputs include but are not limited to the rate of fire spread (ros in $\mathrm{m} / \mathrm{s}$ ), the direction of maximum spread $\left(s d r\right.$ in $\left.^{\circ}\right)$ and reaction intensity $\left(r i\right.$ in $\left.k W / m^{2}\right)$. In accordance with Bachmann (2001), the rate of spread, for example, is formulated as:

$$
\operatorname{ros}=\frac{r i \cdot \xi \cdot\left(1+\Phi_{c}\right)}{\rho_{b} \cdot \varepsilon \cdot Q_{i g}}
$$

where $\xi$ is the propagating flux ratio, $\Phi_{\mathrm{c}}$ is a combination of the slope and wind factors, $\rho_{b}$ is the ovendry bulk density, $\varepsilon$ is the effective heating number, and $\mathrm{Q}_{\mathrm{ig}}$ is the heat of preignition.

The inputs for the model can generally be identified as 12 parameters, which are the ovendry fuel loading $w_{o}$, fuel depth $d$, surface-area-to-volume ratio $s v$, fuel heat content heat, fuel particle density $\rho_{p}$, fuel moisture content $M$, fuel total mineral content $S_{t}$, fuel effective 
mineral $S_{e}$, wind speed at midflame height $w s p$, slope (vertical rise/horizontal run) slp, wind direction $\theta$, and fuel moisture of extinction $m_{x}$. The three parameters $w_{o}, s v$ and $M$ can be further subdivided into five size classes that categorize the different fuel moisture time-lag classes: dead fuel, $0-0.6 \mathrm{~cm}(d 1)$; dead fuel $0.6-2.5 \mathrm{~cm}(d 2)$; dead fuel, $2.5-7.5 \mathrm{~cm}(d 3)$; live herbaceous fuel $(l h)$; and live woody fuel $(l w)$. Hence, the total number of input parameters for the Rothermel model reaches 24 .

Assuming uncertainty exists in all input parameters, we are interested in determining parameters that cause larger variations in the model output. The methodology we will use is described in the following section.

\section{Sobol' Global Sensitivity Analysis}

Sensitivity analysis (SA) attributes the uncertainty in the output to different sources of input variables and uses the amount of contribution of an input to characterize its importance. An input parameter whose variation leads to large uncertainty in the model output is tagged as sensitive or important. Therefore it is of practical importance to identify the sensitive parameters and minimize their uncertainty so that more robust models in support of fire management can be developed.

\section{Variance Decomposition Based on ANOVA-HDMR}

Sobol' Global Sensitivity Analysis (GSA) is based on the ANOVA-high dimensional model representation (ANOVA-HDMR) that decomposes the model as a series of component functions that hierarchically increase in the number of parameters. Suppose we denote the 
model function by $\mathrm{f}(\mathbf{x})$, where $\mathbf{x}$ represents the $d$ input parameters: $\mathrm{x}_{1}, \mathrm{x}_{2}, \ldots, \mathrm{x}_{d}$. Then the ANOVA-HDMR of $f(\mathbf{x})$ is given by

$$
\begin{aligned}
\mathrm{f}(\mathbf{x})=\mathrm{f}_{\varnothing} & +\sum_{i_{1}} f_{\left\{i_{1}\right\}}\left(x_{i_{1}}\right)+\sum_{i_{1}<i_{2}} f_{\left\{i_{1}, i_{2}\right\}}\left(x_{i_{1}}, x_{i_{2}}\right)+\cdots \\
& +\sum_{i_{1}<\cdots<i_{r}} f_{\left\{i_{1}, \cdots, i_{r}\right\}}\left(x_{i_{1}}, \cdots, x_{i_{r}}\right)+\cdots+f_{\{1,2, \ldots, d\}}\left(x_{1}, \cdots, x_{d}\right) .
\end{aligned}
$$

The constant term $\mathrm{f}_{\varnothing}$ is the mean (expectation) of the model, $f_{\left\{i_{1}\right\}}\left(x_{i_{1}}\right)$ represents the main effect of the parameter $\mathrm{x}_{\mathrm{i}_{1}}, f_{\left\{i_{1}, i_{2}\right\}}\left(x_{i_{1}}, x_{i_{2}}\right)$ represents the second order interactions due to $\mathrm{x}_{\mathrm{i}_{1}}$ and $\mathrm{x}_{\mathrm{i}_{2}}$, and so on. Each non-zero component function, $\mathrm{f}_{\mathrm{u}}, \mathrm{u} \subseteq\{1,2, \ldots, \mathrm{d}\}$, has a partial variance $\sigma_{\mathrm{u}}^{2}$. The ANOVA-HDMR is an orthogonal decomposition of the model function in the sense that the total variance of the model is decomposed as the sum of the variances of the component functions, i.e., $\sigma^{2}=\sum_{\mathrm{u} \subseteq\{1,2, \ldots, d\}} \sigma_{\mathrm{u}}^{2}$. The normalized quantity $\sigma_{\mathrm{u}}^{2} / \sigma^{2}$ is used to measure the importance of the parameters related to the index set $\mathrm{u}$.

There is a total of $2^{d}-1$ partial variances and to compute all of them can be impractical. Sobol' (1993) introduces two types of sensitivity measures for a certain parameter $\mathrm{x}_{\mathrm{i}}$ :

$$
\mathrm{S}_{\{\mathrm{i}\}}=\frac{1}{\sigma^{2}} \sigma_{\{\mathrm{i}\}}^{2}, \quad \overline{\mathrm{S}}_{\{\mathrm{i}\}}=\frac{1}{\sigma^{2}} \sum_{\mathrm{i} \in \mathrm{v}} \sigma_{\mathrm{v}}^{2}
$$

$\mathrm{S}$ and $\overline{\mathrm{S}}$ are called the main effect and total effect respectively. The main effect only considers the impact of a parameter to the function output by itself, whereas the total effect incorporates the interactions of the parameter with other parameters. The above definitions can be easily extended to define sensitivity measures for groups of parameters. A Monte Carlo based algorithm to compute the main and total effects has been designed by Sobol' (1993), and improved by Saltelli (2002).

\section{Truncated ANOVA-HDMR and Orthogonal Polynomial Expansion}


In the presence of a large number of input parameters, computing Sobol' sensitivity indices can be time consuming. A remedy is to use a truncated version of ANOVA-HDMR (1) where we only compute a few lower order interactions. Li et al. (2001) suggested expanding the component functions of (1) in terms of series of orthogonal polynomials:

$$
\begin{aligned}
& \text { (2) } \mathrm{f}_{\left\{\mathrm{i}_{1}\right\}}\left(x_{i_{1}}\right)=\sum_{r_{1}=1}^{R_{1}^{1}} \alpha_{r_{1}}^{i_{1}} \phi_{r_{1}}^{i_{1}}\left(x_{i_{1}}\right) \\
& \text { (3) } \mathrm{f}_{\left\{\mathrm{i}_{1}, \mathrm{i}_{2}\right\}}\left(x_{i_{1}}, x_{i_{2}}\right)=\sum_{r_{1}=1}^{R_{1}^{2}} \sum_{r_{2}=1}^{R_{2}^{2}} \beta_{r_{1} r_{2}}^{i_{1} i_{2}} \phi_{r_{1}}^{i_{1}}\left(x_{i_{1}}\right) \phi_{r_{2}}^{i_{2}}\left(x_{i_{2}}\right)
\end{aligned}
$$

as $\mathrm{i}_{1}<\mathrm{i}_{2}<\cdots<\mathrm{i}_{\mathrm{d}^{\prime}}$ take values in the index set $\{1,2, \ldots, \mathrm{d}\}$ and $1 \leq \mathrm{d}^{\prime} \leq \mathrm{d}$. Here

$\phi_{r_{1}}^{i_{1}}\left(x_{i_{1}}\right), \mathrm{r}_{1}=1,2, \ldots$, are orthogonal polynomials of degree $\mathrm{r}_{1}$ associated with the parameter $\mathrm{x}_{\mathrm{i}_{1}}$, and $\alpha_{r_{1}}^{i_{1}}, \beta_{r_{1} r_{2}}^{i_{1} i_{2}}, \ldots$ are the coefficients of expansion.

It is noteworthy that the polynomial expansions for the first and second order components (2) and (3), as well as for the omitted higher order components, conform to the well-known polynomial chaos expansion (PCE) (Xiu and Karniadakis 2002). The clear distinction lies in the fact that PCE in general sets a unified maximum expansion order and all expansion terms do not exceed the specified order, while for the approximation (2) and (3) each component function can have its own maximum expansion degree, i.e., $R_{1}^{1}$ and $R_{1}^{2}+R_{2}^{2}$ can be different.

Due to the orthogonality of the polynomial basis, the expansion coefficients can be computed by orthogonal projections:

$$
\text { (4) } \quad \alpha_{r_{1}}^{i_{1}}=\frac{\int_{\Omega} \mathrm{f}(\mathbf{x}) \phi_{r_{1}}^{i_{1}}\left(x_{i_{1}}\right) p(\boldsymbol{x}) d \boldsymbol{x}}{\int\left(\phi_{r_{1}}^{i_{1}}\left(x_{i_{1}}\right)\right)^{2} p_{i_{1}}\left(x_{i_{1}}\right) d x_{i_{1}}}
$$




$$
\text { (5) } \beta_{r_{1} r_{2}}^{i_{1} i_{2}}=\frac{\int_{\Omega} \mathrm{f}(\mathbf{x}) \phi_{r_{1}}^{i_{1}}\left(x_{i_{1}}\right) \phi_{r_{2}}^{i_{2}}\left(x_{i_{2}}\right) p(\boldsymbol{x}) d \boldsymbol{x}}{\iint\left(\phi_{r_{1}}^{i_{1}}\left(x_{i_{1}}\right) \phi_{r_{2}}^{i_{2}}\left(x_{i_{2}}\right)\right)^{2} p_{i_{1}}\left(x_{i_{1}}\right) p_{i_{2}}\left(x_{i_{2}}\right) d x_{i_{1}} d x_{i_{2}}}
$$

as $\mathrm{i}_{1}<\mathrm{i}_{2}<\cdots<\mathrm{i}_{\mathrm{d}^{\prime}}$ take values in the index set $\{1,2, \ldots, \mathrm{d}\}$ and $1 \leq \mathrm{d}^{\prime} \leq \mathrm{d}$. Here $\boldsymbol{\Omega}$ is the domain of the input parameters and $p(x)=\prod_{i=1}^{d} p_{i}\left(x_{i}\right)$ is the joint probability density function (pdf). If orthonormal polynomials are used as the basis in the expansions (2) and (3), then the denominators will be 1 . The numerators involve high dimensional integrals, which can be approximated by Monte Carlo or quasi-Monte Carlo methods (QMC) (Niederreiter 1992; Caflisch 1998). Only one sequence of random numbers is adequate to compute all the expansion coefficients, as well as the model output variance $\sigma^{2}$. For orthonormal polynomial expansions, the main effects and total effects can consequently be approximated by

$$
\text { (6) } \mathrm{S}_{\{\mathrm{i}\}}=\frac{1}{\sigma^{2}} \sum_{\mathrm{r}_{1}}\left(\alpha_{\mathrm{r}_{1}}^{\mathrm{i}}\right)^{2}, \quad \overline{\mathrm{S}}_{\{\mathrm{i}\}}=\frac{1}{\sigma^{2}}\left(\sum_{\mathrm{r}_{1}}\left(\alpha_{\mathrm{r}_{1}}^{\mathrm{i}}\right)^{2}+\sum_{\mathrm{r}_{1}} \sum_{\mathrm{r}_{2}} \sum_{j}\left(\beta_{r_{1} r_{2}}^{i j}\right)^{2}+\cdots\right) \text {, }
$$

since the inner products (the denominators shown in (4) and (5)) of the polynomial basis are all 1. For general orthogonal polynomials, each term in (6) has to be multiplied by the inner product of its corresponding basis. Extension of (6) for a group of parameters can be made by including the coefficient terms related to the group in the summations.

\section{Sensitivity Analysis of Fuel Models}

We apply Sobol' GSA to the Rothermel model for different types of fuel. Six fuel models are considered: FM1, short grass; FM 3, tall grass; FM4, chaparral; FM9, hardwood litter; FM10, timber; FM11, light logging slash. These fuel models are selected from the 13 standard fuel models (Anderson 1982, Albini 1976), which extend the original 11 standard fuel models specified by Rothermel (1972). 
Of the 24 input parameters of the Rothermel model, 14 parameters are common to all six fuel models. The common parameters are listed in Table 1 . Values of the remaining 10 parameters are unique to the fuel models, and they are shown in Table 2. The symbols that denote the parameters are consistent with those introduced in the second section.

Due to the measurement error in experiments and other factors, uncertainties are assumed to exist in all input parameters. We model the uncertainty in each parameter using the uniform distribution $U(a, b)$, where the constants $a$ and $b$ are determined so that the mean of the distribution is as given in Table 1 and Table 2, and the standard deviation is 5\% of the mean. The purpose of setting the coefficient of variation (defined as the ratio of the standard deviation to the mean) to be $5 \%$ is to ensure that both dead and living fuel damping moistures do not exceed their extinction moistures. In this case, fire propagates on both categories of fuels, which is the case of most interest to fire management.

Note that fuels of certain size classes can be absent for a fuel model. Consequently the model outputs have zero sensitivity to the corresponding surface-area-to-volume ratio and fuel moisture content parameters, since they are only related to the fuel loading parameters in the model formulation. As a result, it makes more sense to consider sensitivity to groups of parameters. The entire set of parameters can be easily categorized into 10 groups as shown in Table 3 .

To compute the Sobol' global sensitivity indices, we employ the second order truncated ANOVA-HDMR, and expand each component function in terms of Legendre polynomials. The highest polynomial expansion orders for the first and second HDMR components are set to be 8 and 5, i.e., $R_{1}^{1}=8$ and $R_{1}^{2}+R_{2}^{2}=5$ in equations (2) and (3). One random-start 
random permuted Halton quasi-random sequence (Ökten 2009) of size $2^{12}$ is generated to compute all expansion coefficients in (4) and (5), in addition to the output variance. All coefficients are then used to construct the Sobol' indices based on the analogous version of (6) that applies to groups of parameters.

\section{Results}

Figure 1 illustrates the total effects of the grouped parameters with respect to the model output ros for different fuel models. In all cases, the indices approximately add up to 1 , meaning that interactions among parameters are very weak, which further validates our employment of a second order truncated ANOVA-HDMR. In view of the fact that total effects take interactions of parameters into consideration, they are tailor-made for identifying insensitive parameters.

The total effects for all the six fuel models considered exhibit some similarities: the parameters $\rho_{p}$, mineral contents, and slp have small total effects. In other words, the impact of variation in these parameters on the output would be insignificant. On the other hand, the sensitivity indices of $d$, heat, and wind are all relatively large, indicating that the variation of ros is highly dependent on the variation of these parameters. Taking light logging slash for example, the three parameters account for about $85 \%$ of the total model variance. Taking control of the uncertainty associated with these parameters is crucial to the prediction of fire behaviors and reduction of fire risk. We also observe that the sensitivity of a parameter varies significantly for different fuel models. For example, the parameter $d$ explains around $40 \%$ of total model variance for light logging slash, but only $12 \%$ for the short grass fuel model. 
The total effects of parameters $m_{x}$ and $s v$ show similarities among all types of fuel except one. For all fuel models except short grass, $m_{x}$ is negligible, while for short grass fuels, $m_{x}$ accounts for $6 \%$ of the total variance, making a nonnegligible impact on the model output. Similar observations can be made for $s v$, which is insignificant for light logging slash, but responsible for around $20 \%$ of total variance in the other fuel models.

The remaining parameters, $\mathrm{m}_{\mathrm{f}}$ and $w 0$, are significant for part of the selected fuel models. For short grass, tall grass, chaparral and timber, $\mathrm{m}_{\mathrm{f}}$ accounts for more than $5 \%$ of the total variance, and hence is considered as a source of uncertainty that cannot be ignored. The parameter $w 0$ is a significant source for the chaparral and timber fuel models. However, it is insignificant for the rest of the models, especially for short grass, hard grass and hardwood litter.

We observe that among the purely landscape/environmental input parameter groups, wind and slope, only wind has a consistently significant impact between $20 \%$ and $40 \%$ of the total variance for the fuel models. The impact of the other environmental parameter groups, fuel loading, fuel moisture, and fuel depth, depends heavily on the fuel model. Finally, among the remaining input parameter groups primarily describing the material, the main two factors contributing to the model variance are heat and surface area to volume ratio. At the highest, these two parameters account for almost $45 \%$ of the total variance in FM9, and at the lowest, $25 \%$ in FM11.

\section{Conclusions}

Parametric uncertainty inescapably exists in wildland fire models, causing difficulty in fire 
behavior prediction. Uncertainty propagates from input parameters to model outputs through fire models, resulting in discrepancies between the model predictions and observations. While quantifying the uncertainty of the model outputs gives the distribution of model predictions, sensitivity analysis identifies which uncertain parameters are responsible for significant model variations. Once the significant parameters are identified, extra resources should be used in order to reduce their uncertainty. This is of vital importance to increase model robustness and reliability, as well as the reinforcement of wildland fire management.

In contrast to local sensitivity analysis which focuses on local rate of change of models, GSA considers variations of parameters in their entire uncertainty range, and therefore provides more informative and reliable results. We applied Sobol's variance-based GSA to the Rothermel model with six standard fuel models. To reduce the computational complexity of Sobol' sensitivity indices, a truncated ANOVA-HDMR approach with orthogonal polynomial expansion was employed. The results show that there are some parameters which are uniformly significant or insignificant for all fuel models. There are also parameters whose importance varies with different fuel models. In applications, these parameters need to be treated differently according to the type of fuel model. If a parameter is significant, then additional resources should be used to mitigate its uncertainty. If a parameter is insignificant, then it can be fixed at its mean value without affecting the total model variance. 


\section{References}

Albini, F.A. 1976. Estimating wildfire behavior and effects. USDA Forest Service, Intermountain Forest and Experiment Station General Technical Report INT-30.

Albini, F.A. 1986. Wildland fire spread by radiation - a model including fuel cooling by natural convection. Combustion Science and Technology 45, 101-113.

Anderson, H.E. 1982. Aids to determining fuel models for estimating fire behavior. USDA Forest Service, Intermountain Forest and Range Experiment Station General Technical Report INT-122. (Ogden, UT)

Andrews, P.L. 2007. BehavePlus fire modeling system: Past, present and future. In 'Proceedings of 7th Symposium on Fire and Forest Meteorology’. 23-25 October 2007, Bar Harbor, ME. (American Meteorological Society: Boston, MA)

Bachmann, A. 2001. GIS-based wildland fire risk analysis. PhD thesis, Universität Zürich, Switzerland.

Caflisch, R.E. 1998. Monte Carlo and quasi-Monte Carlo methods. Acta Numerica, 1-49.

Catchpole, E., Catchpole, W. 1991. Modelling moisture damping for fire spread in a mixture of live and dead fuels. International Journal of Wildland Fire 1, 101-106.

Clark, T.L. 2004. Description of a coupled atmosphere-fire model. International Journal of Wildland Fire 13, 49-63.

Finney, M.A. 2004. FARSITE: Fire area simulator-model development and evaluation. USDA Forest Service, Rocky Mountain Research Station Research Paper RMRS-RP-4 Revised. (Ogden, UT)

Jimenez, E., Hussaini, M.Y., Goodrick, S. 2007. Uncertainty quantification in Rothermel's model using an efficient sampling method. In 'Proceedings of the $2^{\text {nd }}$ Fire Behavior and Fuels Conference'. 26-30 March 2007, Destin, FL. (International Association of Wildland 
Fire: Missoula, MT)

Jimenez, E., Hussaini, M.Y., Goodrick, S. 2008. Quantifying parametric uncertainty in the Rothermel model. International Journal of Wildland Fire 17, 638-649.

Li, G., Rosenthal, C., Rabitz, H. 2001. High dimensional model representations. Journal of Physical Chemistry A 105, 7765-7777.

Linn, R.R., Reisner, J., Colman, J., Winterkamp, J. 2002. Studying wildfire using FIRETEC. International Journal of Wildland Fire 11, 1-14.

Liu, Y., Jimenez, E., Hussaini, M.Y., Ökten, G., Goodrick, S. 2015. Parametric uncertainty quantification in the Rothermel model with randomized quasi-Monte Carlo methods. International Journal of Wildland Fire 24, 307-316.

McMaster, A.W. 1973. A statistical fire spread model for forest fires. Technical Report, No. 14. Department of Statistics, University of California, Riverside, California.

Niederreiter, H. 1992. Random Number Generation and Quasi-Monte Carlo Methods. Society for Industrial and Applied Mathematics, Philadelphia, PA.

Ökten, G. 2009. Generalized von Neumann-Kakutani transformation and random-start scrambled Halton sequences. Journal of Complexity 25, 318-331.

Pyne, S.J., Andrews, P.L., Laven, R.D. 1996. Introduction to Wildland Fire. JohnWiley \& Sons, New York.

Rabitz, H., Alış, Ö., Shorter, J., Shim, K. 1999. Efficient input-output model representations. Computer Physics Communications 117, 11-20.

Rothermel, R.C. 1972. A mathematical model for predicting fire spread in wildland fuels. USDA Forest Service, Intermountain Forest and Range Experiment Station Research Paper INT-115. (Ogden, UT)

Saltelli, A. 2002. Making best use of model evaluations to compute sensitivity indices. Computer Physics Communications 145, 280-297. 
Salvador, R., Pinol, J., Tarantola, S., Pla, E. 2001. Global sensitivity analysis and scale effects of a fire propagation model used over Mediterranean shrublands. Ecological Modelling 136, 175-189.

Sandberg, D.V., Riccardi, C.L., Schaaf, M.D. 2007. Reformulation of Rothermel's wildland fire behaviour model for heterogeneous fuelbeds. Canadian Journal of Forest Research 37, $2438-2455$.

Sobol', I. 1993. Sensitivity estimates for non-linear mathematical models. Mathematical Modeling and Computational Experiment 1, 407-414.

Sobol', I. 2001. Global sensitivity indices for nonlinear mathematical models and their Monte Carlo estimates. Mathematics and Computers in Simulation 55, 271-280.

Wilson, R. A. 1990. Reexamination of Rothermel's fire spread equations in no-wind and noslope conditions. USDA Forest Service, Wildfires, Paper 8. (Ogden, UT)

Xiu, D., Karniadakis, G.E. 2002. The Wiener-Askey polynomial chaos for stochastic differential equations. SIAM Journal on Scientific Computing 24, 619-644. 
Table 1-Common fuel model parameters.

\begin{tabular}{lccc}
\hline \multicolumn{1}{c}{ Parameter } & Symbol & Value & Unit \\
\hline Low heat content & heat & 18622.0 & $\mathrm{~kJ} / \mathrm{kg}$ \\
1-h fuel moisture & $m_{d 1}$ & 8.0 & $\%$ \\
10-h fuel moisture & $m_{d 2}$ & 8.0 & $\%$ \\
100-h fuel moisture & $m_{d 3}$ & 8.0 & $\%$ \\
Live herbaceous fuel moisture & $m_{l h}$ & 150.0 & $\%$ \\
Live woody fuel moisture & $m_{l w}$ & 150.0 & $\%$ \\
Particle density & $\rho_{p}$ & 512.5 & $\mathrm{~kg} / \mathrm{m}^{3}$ \\
Effective mineral content & $S_{e}$ & 1.0 & $\%$ \\
Slope & $s l p$ & 14.04 & $\mathrm{o}$ \\
Total mineral content & $S_{t}$ & 5.55 & $\mathrm{~m} / \mathrm{s}$ \\
10-h surface area/vol ratio & $s v_{d 2}$ & 358.0 & $\mathrm{~m}$ \\
100-h surface area/vol ratio & $s v_{d 3}$ & 98.0 & $\mathrm{~m}^{2} / \mathrm{m}^{3}$ \\
Direction of wind & $\theta$ & 45 & $\mathrm{o}$ \\
Midflame wind speed & $w s p$ & 2.3 & $\%$ \\
\hline & & & \\
\hline
\end{tabular}


Table 2-Input parameter values unique to fuel models.

\begin{tabular}{cccccccc}
\hline Parameter & FM1 & FM3 & FM4 & FM9 & FM10 & FM11 & Unit \\
\hline$d$ & 0.3 & 0.76 & 1.83 & 0.06 & 0.3 & 0.3 & $\mathrm{~m}$ \\
$m_{x}$ & 12 & 25 & 20 & 25 & 25 & 15 & $\%$ \\
$s v_{d 1}$ & 11483.0 & 4921.3 & 6562.0 & 8202.0 & 6562.0 & 4921.0 & $\mathrm{~m}^{2} / \mathrm{m}^{3}$ \\
$s v_{l h}$ & 4921.0 & 4921.0 & 4921.0 & 4921.0 & 4921.0 & 4921.0 & $\mathrm{~m}^{2} / \mathrm{m}^{3}$ \\
$s v_{l w}$ & 4921.0 & 4921.0 & 4921.0 & 4921.0 & 4921.0 & 4921.0 & $\mathrm{~m}^{2} / \mathrm{m}^{3}$ \\
$w 0_{d 1}$ & 0.17 & 0.67 & 1.12 & 0.65 & 0.67 & 0.34 & $\mathrm{~kg} / \mathrm{m}^{2}$ \\
$w 0_{d 2}$ & 0 & 0 & 0.90 & 0.09 & 0.45 & 1.01 & $\mathrm{~kg} / \mathrm{m}^{2}$ \\
$w 0_{d 3}$ & 0 & 0 & 0.45 & 0.03 & 1.12 & 1.23 & $\mathrm{~kg} / \mathrm{m}^{2}$ \\
$w 0_{l h}$ & 0 & 0 & 0 & 0 & 0 & 0 & $\mathrm{~kg} / \mathrm{m}^{2}$ \\
$w 0_{l w}$ & 0 & 0 & 1.12 & 0 & 0.45 & 0 & $\mathrm{~kg} / \mathrm{m}^{2}$ \\
\hline
\end{tabular}

Table 3 - Grouping of input parameters.

\begin{tabular}{|c|c|c|c|c|c|c|c|c|c|}
\hline Wind & Slope & $\begin{array}{c}\text { Fuel } \\
\text { loading }\end{array}$ & $\begin{array}{c}\text { Fuel } \\
\text { moisture } \\
\text { content }\end{array}$ & $\begin{array}{l}\text { Fuel } \\
\text { depth }\end{array}$ & $\begin{array}{c}\text { Low } \\
\text { heat } \\
\text { content }\end{array}$ & $\begin{array}{l}\text { Moisture } \\
\text { of } \\
\text { extinction }\end{array}$ & $\begin{array}{l}\text { Particle } \\
\text { density }\end{array}$ & $\begin{array}{l}\text { Mineral } \\
\text { contents }\end{array}$ & $\begin{array}{l}\text { Surface- } \\
\text { area-to- } \\
\text { volume } \\
\text { ratio }\end{array}$ \\
\hline $\begin{array}{c}\Theta \\
w s p\end{array}$ & slp & $\begin{array}{l}w 0_{d 1} \\
w 0_{d 2} \\
w 0_{d 3} \\
w 0_{l h} \\
w 0_{l w}\end{array}$ & $\begin{array}{c}m_{d 1} m_{d 2} \\
m_{d 3} m_{l h} \\
m_{l w}\end{array}$ & $d$ & heat & $m_{x}$ & $\rho_{p}$ & $\begin{array}{l}S_{e} \\
S_{t}\end{array}$ & $\begin{array}{l}s v_{d 1} \\
s v_{d 2} \\
s v_{d 3} \\
s v_{l h} \\
s v_{l w}\end{array}$ \\
\hline
\end{tabular}




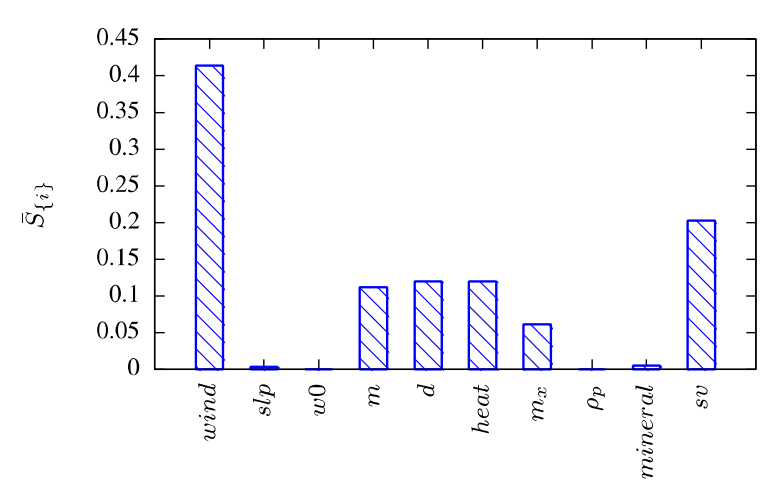

Parameters

(a) FM1

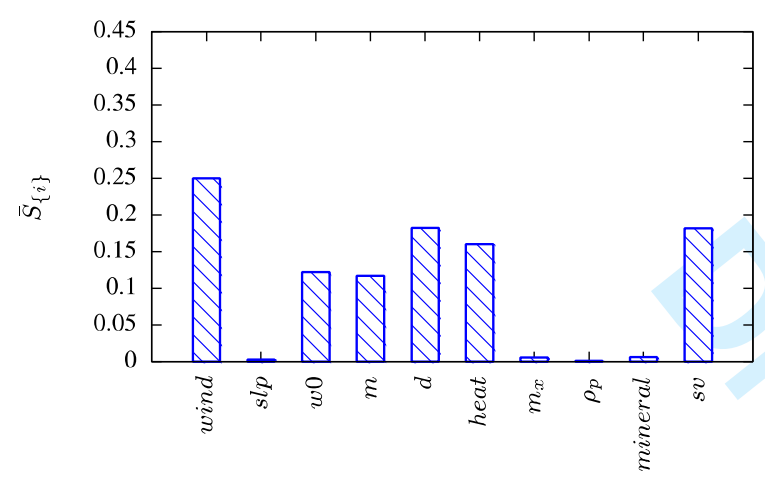

Parameters

(c) FM4

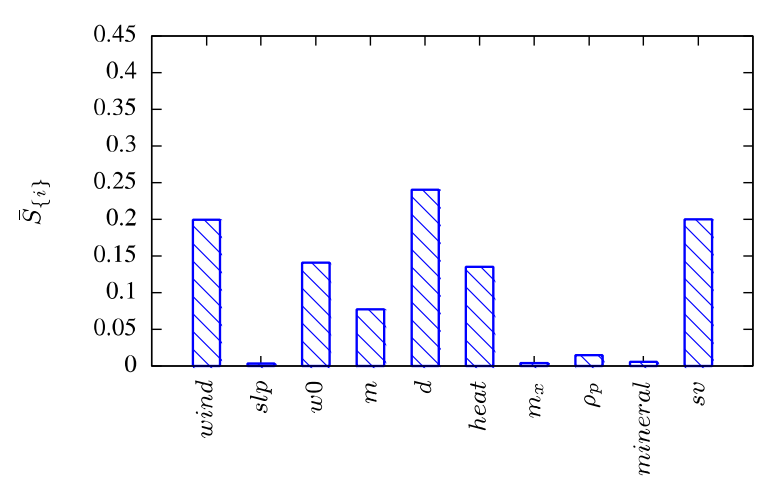

Parameters

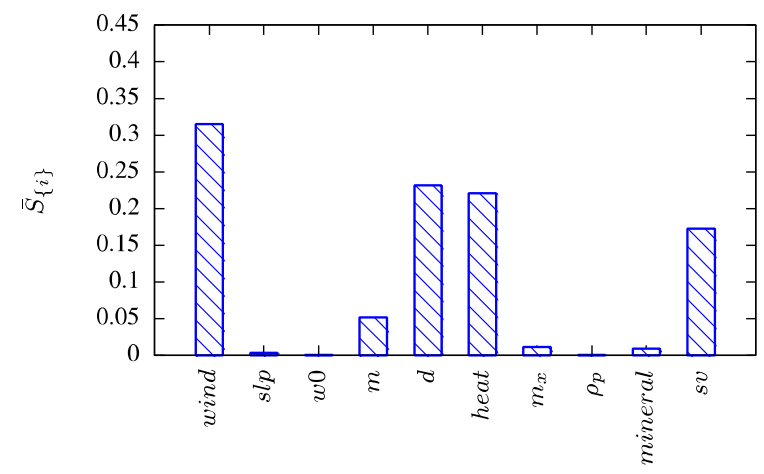

Parameters

(b) FM3

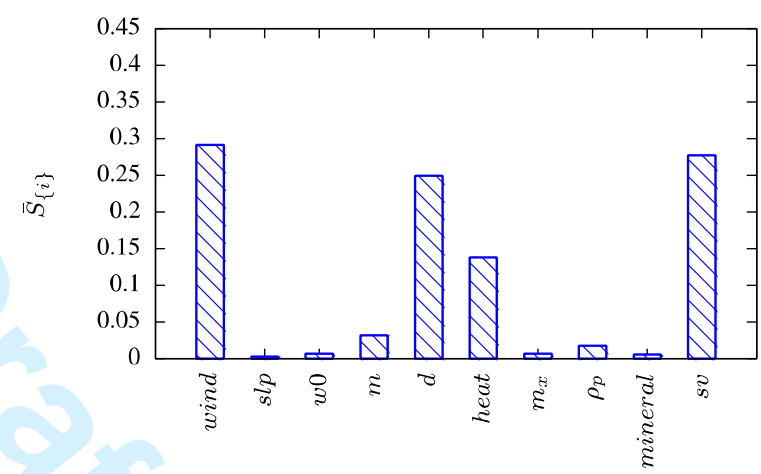

Parameters

(d) FM9

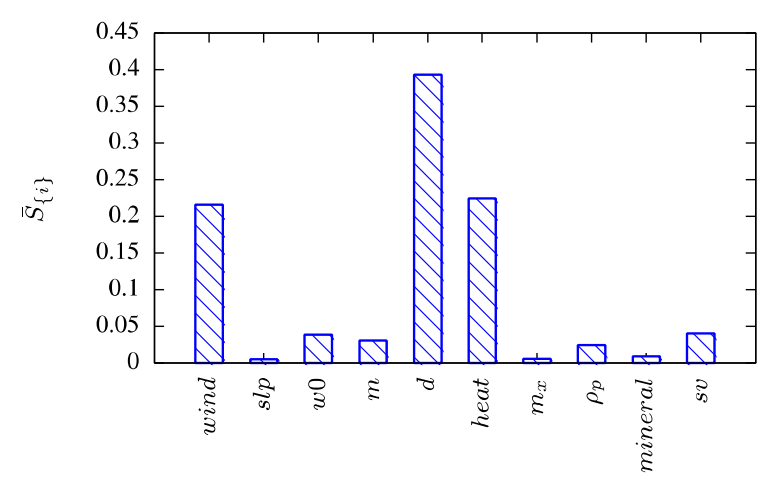

Parameters

(e) FM10

(f) FM11

Figure 1-Sobol' global sensitivity indices (total effects) for the Rothermel model with various fuel types. The output is ros. The indices are obtained by approximating the 2 nd order truncated ANOVA-HDMR with a Legendre polynomial expansion with coefficients computed by QMC. 